\title{
New habits in branched polyethylene single crystals
}

\author{
J.F. Vega, ${ }^{\mathrm{a}^{*}}$ A. J. Müller ${ }^{\mathrm{b}, \mathrm{c}}$ and J. Martínez-Salazar ${ }^{\mathrm{a}}$ \\ ${ }^{a}$ Biophym, Departamento de Física Macromolecular, Instituto de Estructura de la Materia (IEM-CSIC) c/Serrano 113 bis, 28006 Madrid, Spain \\ ${ }^{b}$ POLYMAT and Polymer Science and Technology Department, Faculty of Chemistry, University of the Basque Country UPV/EHU, Paseo \\ Manuel de Lardizabal 3, 20018 Donostia-San Sebastián, Spain \\ 'IKERBASQUE, Basque Foundation for Science, Bilbao, Spain \\ *corresponding author: jf.vega@csic.es
}

\begin{abstract}
Faceted polymer single crystals obtained from solution have been examined for decades. Most of the studies have been performed with linear polyethylene. It is well known that solution-grown linear polyethylene single crystals may exhibit different and often complex morphologies and habits. However, the effect of molecular architecture (i.e., short chain branching) in the morphology of the single crystals remains practically unexplored. At the highest crystallization temperature investigated, the shape of the crystals is lozenge-like, but with curved and slightly truncated $\{110\}$ faces. As the crystallization temperature decreases, strong changes in the width-to-length ratio and, consequently in the characteristic angles of the single crystal occur. Interestingly, the single crystals obtained at the lowest crystallization temperature explored exhibit a nearly square shape. This phenomenon has not yet been observed in linear high molecular weight polyethylene, for which the characteristic lozenge habit with straight $\{110\}$ faces is retained as crystallization temperature decreases. The application of the Shcherbina and Ungar approach to fit the unusual shapes of the branched PE single crystals obtained in this work requires a strong decrease in the ratio of the rates of propagation to the right and to the left of the growth edge. This result is probably linked to different steric conditions of chain attachments into non-equivalent niches induced by the presence of branches.
\end{abstract}

Keywords: Single Crystals; Habits; Polyethylene; Short Chain Branching; Curved Faces

\section{Introduction}

The variations in shape, morphology and structure of single crystals, particularly of linear PE, have been intensively studied for more than 50 years, but renewed interest in the study of linear PE single crystals in the last decade has appeared with the works of Organ et al. [1], Tian et al. [2], Nakamura et al. [3] and Zhang et al. [4].

A particularly striking phenomenon is the occasional appearance of curved habits. Solution-grown crystals of polyethylene (PE) [5], among other polymers, such as polyethylene oxide (PEO) [6], poly(vinylidene fluoride) (PVDF) [7] and poly-e-caprolactone (PCL) [8] can exhibit curved habits. In linear PE single crystals, curved $\{100\}$ faces are seen at low supercooling $(\Delta T)$, as the lozenge crystals $\{110\}$ faces are truncated by the slow growth of curved $\{100\}$ faces. At lower $\Delta T$, elongated lamellae along the b-axis are observed, resembling melt-grown crystals [9].

In the case of long n-alkanes a similar shape classification is seen when they are crystallized in noctacosane, but upon increasing $\Delta \mathrm{T}$. However, when they are crystallized in 1-phenyldecane, crystal lamellae exclusively bounded by $\{110\}$ lateral faces are obtained at low $\Delta T$, but in this case the lenticular shape extends along the a-axis rather than the $b$-axis $[10,11]$.

It has been shown by Ungar et al., that crystal faces are curved when the propagation rate $(\mathrm{v})$ is very small [12]. This low rate is due to "self-poisoning" or "pinning" effect of incorrect chain attachment. This has been demonstrated in the extreme case of $\{100\}$ face growth rate minima in long-chain alkanes. This phenomenon turned out to be evident in monodisperse long-chain alkanes due to the quantized character of lamellar thickness ("integer folding"). However, Ungar et al. have suggested that there are strong experimental indications that "self-poisoning" also plays an important role in crystallization of high molecular weight $\mathrm{PE}$, particularly at higher temperatures. In these conditions the $\{100\}$ face growth impediment is most pronounced, suggesting that the "self-poisoning" increases with temperature. This is possible since for a given $T_{c}$, there are crystal stems shorter than the minimum stable fold length $\left(I_{\min } \propto \Delta \mathrm{T}^{-1}\right)$, but with lifetime high enough to cause growth obstruction as well as a higher attachment rate because of a lower energy barrier.

As far as the authors are aware, curved $\{110\}$ faces in high molecular weight PE have not been observed until recently. In a previous work, the effect of short chain branching (SCB) on the lateral habits of PE single crystals at a constant $\Delta T=30^{\circ} \mathrm{C}$ were explored [13]. It was shown that SCB causes a strong effect in both the morphology and the structure of single crystals. The most salient feature observed was the curvature in $\{110\}$ faces. It was also shown that the presence of SCB causes the curvature of $\{110\}$ faces, opening up the possibility of studying this fascinating phenomenon in high molecular weight PE. 
The curvature observed in PE single crystals is related to the asymmetric growth of the $\{110\}$ faces and it can be explained in the context of Shcherbina-Ungar (SU) approach [14-16], by a continuous increase in the step initiation (i) - step propagation (v) rates ratio with SCB, i.e., nucleation events are favoured against stem propagation by the presence of chain defects. Additionally, the SU model suggests that the key to understand the variable curvature of $\{110\}$ faces is to assume that the rates of propagation to the right $\left(v_{r}\right)$ and to the left $\left(v_{1}\right)$ of the growth front are different (Fig. 1).

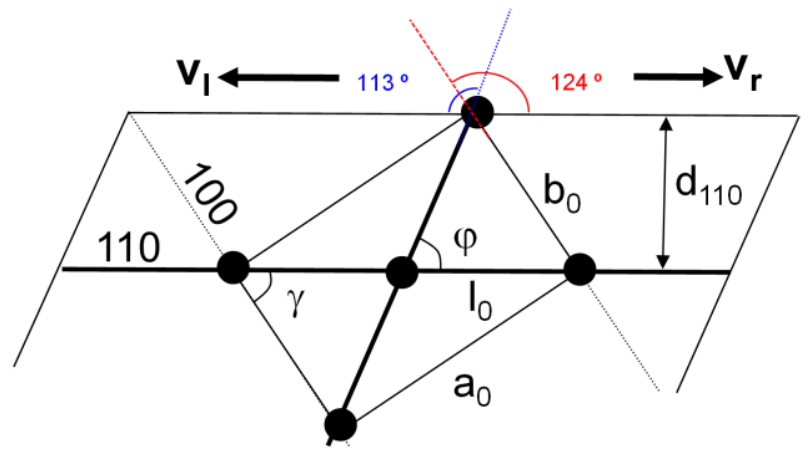

$\overline{110}$

Fig. 1. Lattice parameters, interchain distances and inter-planar angles for orthorhombic PE (adapted from references [14-16]).

In the case of branched PE, and for the conditions selected in a previous study $\left(\Delta T=30{ }^{\circ} \mathrm{C}\right)$ with a similar material, the ratio $v_{r} / v_{l}$ is close to unity. In this communication, the investigation is extended to a broad range supercooling degrees $\left(\Delta T=30-45{ }^{\circ} \mathrm{C}\right)$ for a selected model homogeneous branched PE sample with $\sim 0.5$ branches every 100 carbon atoms (PE05). The objective is to detect changes in $V_{r} / v_{1}$ ratio that explain the new habits observed in single crystals of branched PE.

\section{Experimental}

The material studied was obtained by polymerization with a single-site metallocene catalyst and supplied by Repsol (Móstoles, Spain). The sample is a poly(ethylene-co-1-hexene) with high molecular weight $\left(\mathrm{M}_{\mathrm{w}}=115 \mathrm{~kg} \cdot \mathrm{mol}^{-1}\right)$, narrow molar mass distribution $\left(M_{w} / M_{n}=2\right)$ and homogeneous distribution of comonomer. The molecular and physical properties were obtained by ${ }^{13} \mathrm{C}$ - Nuclear Magnetic Resonance, Size Exclusion Chromatography and CRYSTAF-TREF analysis. Please refer to previous works for details about molecular and physical characterization [13,1722].

A self-seeding procedure was employed before the samples were isothermally crystallized from solution. A solution of the polymer in $p$-xylene was prepared with a concentration of $0.02 \%(\mathrm{w} / \mathrm{w})$, which corresponds to a concentration well below $c^{*}$, i.e., the coil overlap concentration. Samples were heated to $120{ }^{\circ} \mathrm{C}$ and cooled within a few minutes to a temperature $T_{c i}=65$ ${ }^{\circ} \mathrm{C}$, where a fast and exhaustive crystallisation took place in a short period of time (15 - 20 minutes). Then the suspensions were slowly heated to a self-seeding temperature of $T_{s}=94.0{ }^{\circ} \mathrm{C}$. The solutions were then recrystallised to obtain single crystals at four temperatures $\left(\mathrm{T}_{\mathrm{c}}=73{ }^{\circ} \mathrm{C}, 68^{\circ} \mathrm{C}, 63^{\circ} \mathrm{C}\right.$ and $\left.58{ }^{\circ} \mathrm{C}\right)$ below $T_{s}$ during 24 hours. At this degree of supercooling almost the whole material crystallizes in 1,2,4 trichlorobenzene during the CRYSTAF experiments (2-3 hours) at a cooling rate of $0.1^{\circ} \mathrm{C} \cdot \mathrm{min}^{-1}$ [13]. Then, it is expected that the isothermal crystallization experiments performed in p-xylene during 24 hours avoided fractionation of the sample and depleted almost the whole dissolved material.

It should be noted that intrachain heterogeneity may also cause fractionation, as a distribution of crystallisable sequences may lead to a broader crystal size distribution. Mirabella reported that the linear chain sequences within homogeneous copolymers with intrachain heterogeneity undergo primary crystallisation from the melt, which produces a population of thick lamellae, thus creating a network that causes constraints on segmental transport for subsequent secondary crystallisation, leading to thinner crystals [23]. Fractionation and, consequently, multimodal melting endotherms have been observed after isothermal crystallization from the melt of such homogeneous copolymers. However, in the case of dilute solution the segmental transport is facilitated, which gives rise to a more homogeneous crystalline structure and monomodal endotherms than in melt crystallised samples [13].

The crystals dispersed in solution were deposited onto carbon-coated grids and shadowed with carbon at an angle of $60^{\circ}$ for their examination by Transmission Electron Microscopy (TEM). A JEOL JEM-2100 transmission electron microscope operated at $200 \mathrm{kV}$ and a nominal magnification of 20,000 was used for bright field image study.

\section{Results and discussion}

The nature of the $\{110\}$ faces found for PE05 single crystals under different crystallisation conditions is examined in detail in Fig. 2. The ability of the PE05 sample to form single crystals is noteworthy. Fig. 2 shows the remarkable effect of $\Delta \mathrm{T}$ on single crystal habits. The single crystals seem to have serrated edges. This can occur due to post-crystallisation and edges growth during cooling from $T_{c}$ to room temperature and evaporation of the solvent. At such low temperatures, small portions of $\{110\}$ faceted lozenges should be visible. However, higher resolution images do not show this characteristic in any of the samples. Moreover, this occurs even at the highest supercoolings 
$\left(\Delta T>35^{\circ} \mathrm{C}\right)$, for which the whole material has already crystallised. In spite of the irregular edges observed in single crystals, an analysis of the curved faces is possible.

The curved $\{110\}$ faces is the one of the more prominent features of the single crystals obtained. As far as we know curved $\{110\}$ faces have not been observed in high molecular weight linear PE crystals until recently by us [13]. The curvature of $\{110\}$ faces is hardly seen in linear $P E$ at high $T_{c}$, i.e., when the propagation rate $(\mathrm{v})$ is small as compared to nucleation rate (i), since in these conditions the crystals are truncated due to the slow growth of $\{100\}$ faces [5]. However, in the present case, propagation becomes delayed due to the presence of SCB that act as chain defects.
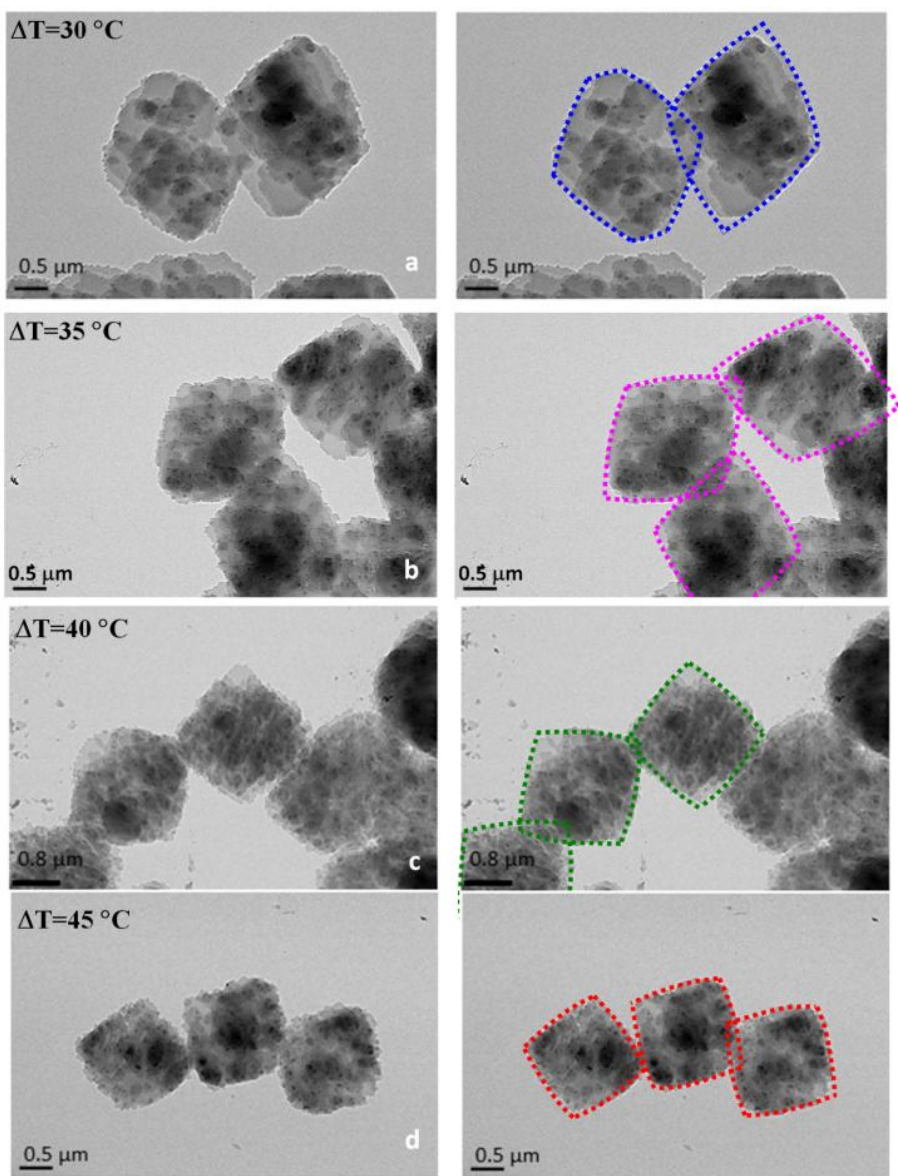

Fig. 2. TEM micrographs of PE05 single crystals (left) and their calculated shapes represented as dotted outlines in selected crystals (right). The dimensionless kinetic parameters are indicated in the $\left(\mathrm{il}_{0}^{2} /\left(\mathrm{v}_{\mathrm{r}}-\mathrm{v}_{\mathrm{I}}\right): \mathrm{v}_{\mathrm{r}} / \mathrm{v}_{\mathrm{l}}\right)$ format for $\{110\}$ faces. (a) $\mathrm{T}_{\mathrm{c}}=73^{\circ} \mathrm{C}(0.09: 1)$; one of the shapes has been calculated with truncated $\{110\}$ faces by $\{100\}$ faces $\left(\mathrm{ib}_{0}{ }^{2} / 2 \mathrm{v}=0.1\right.$, reference [12]); (b) $\mathrm{T}_{\mathrm{c}}=68{ }^{\circ} \mathrm{C}(0.075: 0.8) ;$ (c) $\mathrm{T}_{\mathrm{c}}=$ $63^{\circ} \mathrm{C}(0.065: 0.5) ;$ (d) $\mathrm{T}_{\mathrm{c}}=58{ }^{\circ} \mathrm{C}(0.05: 0.3)$.

For comparison purposes we have included as Supplementary Information micrographs of the single crystals obtained in the same experimental conditions for a linear PE sample of similar $M_{w}$ and $M_{w} / M_{n}$ [13]. In this case, and as it is expected, lozenge crystals are observed, with slightly truncated $\{100\}$ faces as supercooling decreases. At the highest supercooling, the crystallisation process changes from nucleation controlled to diffusion limited and the habit becomes dendritic, as it was reported by Toda and Kiho [24].

The truncation of $\{110\}$ by $\{100\}$ faces is also observed, at least for low supercoolings (see micrographs in Fig. 2a and 2b), but in a lesser extent than in the case of linear PE. In the case of linear PE, a decrease in $T_{c}$ directly leads to the formation of lozenge faceted single crystals with straight edges. In that case, the width-to-length ratio of the single crystal is $\mathrm{I}_{\mathrm{b}} / \mathrm{l}_{\mathrm{a}}=\tan$

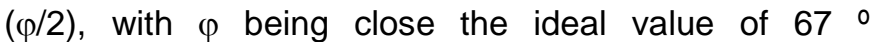
calculated from the unit cell dimensions [5]. This is not the case of the branched PE05 sample studied here. The curvature of $\{110\}$ faces seems to slightly decrease with increasing $\Delta \mathrm{T}$. This suggests that a "self-poisoning" or "pinning" mechanism may be responsible. If such a mechanism is present in high molecular weight branched PE, it should increase with $T_{c}$, as suggested by Putra and Ungar in linear PE $[11,12]$.

The results presented in this work suggest that the presence of branches is responsible for the production of the new single crystal morphologies reported here. SCB induce the change to curved crystal habits, due to the confinement effect of the comonomer at the lowest $T_{c}$ values. The branches are efficient enough to obstruct the face crystal growth. Additionally, the effective crystallisable sequence becomes even shorter, as the branches are not able to enter the crystal lattice. This makes the propagation rate (v) somehow smaller than in linear PE at a given supercooling, making incorrect chain attachment more probable.

More striking is the strong increase observed in the width-to-length ratio $\left(\mathrm{l}_{\mathrm{b}} / \mathrm{l}_{\mathrm{a}}\right)$ of the single crystals with $\Delta T$ in Fig. 2, which is parallel to an increase in the angle $\varphi$ of the acute apex above the crystallographic value of $67^{\circ}$, to values higher than $90^{\circ}$ (see Table 1 ). The increase of this angle has been attributed to the appearance of submicroscopic $\{100\}$ sectors acting as slow-growing substrate for $\{110\}$ faces in long $n$-alkanes [10].

It was shown by Mansfield [25], Point and Villers [26], and Toda [27] that the symmetric curvature of $\{100\}$ faces can be explained quantitatively by applying the Seto and Frank model $[28,29]$. If the ratio of nucleation (i) and step propagation (v) is small, a flat face is formed; an increase in this ratio with an increase of $T_{c}$ leads to a curvature of the $\{100\}$ growth front. On the other hand, Ungar and Putra found in long $n$ alkanes just the opposite; a-axis lenticular habits in monodisperse long alkanes as $T_{c}$ decreases $[10,11]$. This observation revealed that $\{110\}$ faces may show a dichotomy of asymmetric curvatures, depending on the propagation ratio in the two opposite directions of the stem layer $\left(v_{r}\right.$ and $\left.v_{l}\right)$. 
Table 1

Fitted shapes of the single crystals obtained at different crystallisation temperatures, dimensionless parameters, il ${ }^{2} /\left(\mathrm{v}_{\mathrm{r}}+\mathrm{v}_{\mathrm{l}}\right)$ and $\mathrm{V}_{\mathrm{r}} / \mathrm{V}_{\mathrm{l}}$, obtained from the fit of the actual shapes of the single crystals to the Shcherbina and Ungar approach, and main calculated morphological characteristic of the single crystals.

\begin{tabular}{|c|c|c|c|c|c|c|}
\hline Shape & $T_{c}$ & $\mathrm{il}_{0}^{2} /\left(\mathrm{v}_{\mathrm{r}}+\mathrm{v}_{\mathrm{l}}\right)$ & $\mathrm{v}_{\mathrm{r}} / \mathbf{v}_{\mathrm{I}}$ & $I_{b} / I_{a}$ & $\gamma$ & $\varphi$ \\
\hline & $68^{\circ} \mathrm{C}$ & 0.090 & 1 & 0.70 & $61.5^{\circ}$ & $87^{\circ}$ \\
\hline & $63^{\circ} \mathrm{C}$ & 0.075 & 0.8 & 0.76 & $59.5^{\circ}$ & $93^{\circ}$ \\
\hline & $58^{\circ} \mathrm{C}$ & 0.065 & 0.5 & 0.88 & $54.5^{\circ}$ & $99^{\circ}$ \\
\hline & $53^{\circ} \mathrm{C}$ & 0.050 & 0.3 & 1.0 & $49.5^{\circ}$ & $107^{\circ}$ \\
\hline
\end{tabular}

The mathematical analysis of the SU model was applied to the curvature of asymmetric $\{110\}$ faces [14$16]$. In this approach the profile of the growth front is given by the equation:

$\left[\frac{X+\frac{v_{l}-v_{r}}{2}}{\frac{v_{l}+v_{r}}{2}}\right]^{2}+\left[\frac{Y}{b \sqrt{i\left(v_{r}+v_{l}\right)}}\right]^{2}=1$

where $X$ and $Y$ are time-independent coordinates on a square lattice. In order to apply Eq. 1 to $\{110\}$ faces in $\mathrm{PE}$, it has to be transposed onto an oblique lattice. For the two branches of the growth front from either side of the maximum at $\mathrm{X}=\left(\mathrm{v}_{\mathrm{r}}-\mathrm{v}_{\mathrm{l}}\right) / 2$, the corrected coordinate $X_{o b}$ is given by:

$X_{o b}=X-\left[d_{110} \sqrt{i\left(v_{r}+v_{l}\right)}-Y_{o b}\right] \cot \varphi$ for $X<\frac{\mathbf{v}_{\mathbf{r}}+\mathbf{v}_{\mathbf{l}}}{2}$

$X_{o b}=X+\left[d_{110} \sqrt{i\left(v_{r}+v_{l}\right)}-Y_{o b}\right] \cot Y$ for $X \geq \frac{v_{r}+v_{l}}{2}$

where $Y_{o b}=\left(d_{110} / l_{0}\right) Y, d_{110}$ the $\{110\}$ interplanar spacing and $I_{0}$ the interchain distance in the crystallographic growth plane, i.e., $\mathrm{I}_{0}=\left[\left(\mathrm{a}_{0}^{2}+\mathrm{b}_{0}{ }^{2}\right)^{1 / 2}\right] / 2$, with $\mathrm{a}_{0}=7.44 \AA$ and $b_{0}=4.97 \AA$ as the lattice parameters. The corresponding inter-planar angles of the orthorhombic crystal lattice are $\varphi=67.6^{\circ}$ and $\gamma=56.3^{\circ}$ (see Fig. 1 for details). The characteristic lattice parameters of the polymeric single crystals obtained by WAXS have been obtained from a previous study with the same material [13]. To illustrate the model the shape of the single crystals depicted in Fig. 3 has been calculated. According to the results of the model in Fig. 3 , as $\mathrm{il}_{0}^{2} /\left(\mathrm{v}_{\mathrm{r}}+\mathrm{v}_{\mathrm{I}}\right)$ ratio decreases, the $\{110\}$ faces become less curved and the obtuse apex angle $(2 \gamma)$ diminishes (see Table 1).

Remarkably, a decrease in $v_{r} / v_{1}$ ratio gives rise to a very important change in the shape of the crystals. The value of the acute angle $\varphi$ is found to increase above the crystallographic value of $67^{\circ}$ with increasing degree of supercooling, a trend that is accompanied by a deviation of $\mathrm{I}_{\mathrm{b}} / \mathrm{I}_{\mathrm{a}}$ from the ideal value of $0.662=\tan (\varphi / 2)$. The application of the model to the results obtained here points mainly to a decrease of $v_{r} / v_{l}$ ratio as $T_{c}$ decreases (i.e., $\Delta \mathrm{T}$ increases).
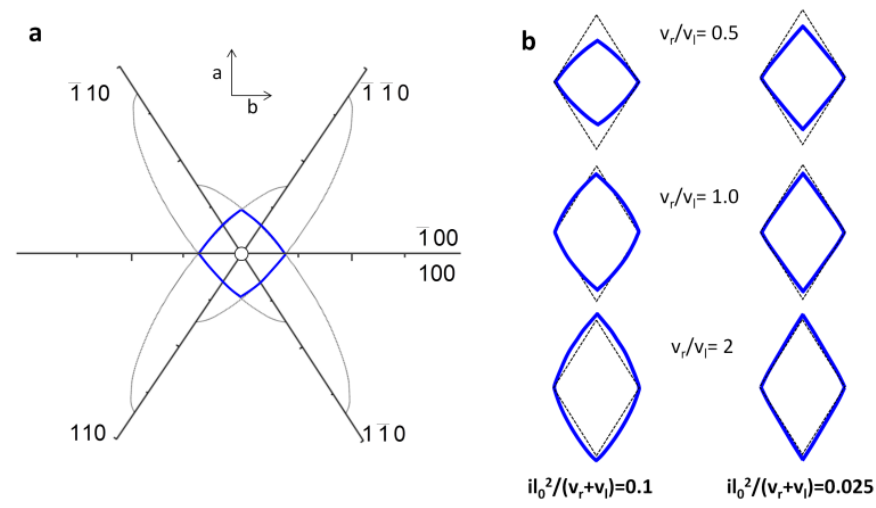

Fig. 3. Construction of crystal shapes bounded by curved faces (PE lattice parameters reported in reference [12] were used in the calculations). (a) The curved growth profiles have been calculated using Eqs. 1 and 2 with the following parameters: $\mathrm{il}_{0}^{2} /\left(\mathrm{v}_{\mathrm{r}}+\mathrm{v}_{1}\right)=0.05$ and $\mathrm{V}_{\mathrm{r}} / \mathrm{V}_{\mathrm{I}}=0.5$ for $\{110\}$ faces. Non-truncated $\{110\}$ faces are considered. Bold blue lines are growth fronts intersecting at crystal corners; (b) Chart of constructed shapes of PE single crystals (in blue) bounded by $\{110\}$ faces. The columns are shapes with the same initiation /propagation ratio $\mathrm{il}_{0}^{2} /\left(\mathrm{v}_{\mathrm{r}}+\mathrm{v}_{1}\right)$; the rows are shapes with the same right and left propagation rate ratio, $\mathrm{V}_{\mathrm{r}} / \mathrm{V}_{\mathrm{l}}$. The corresponding lozenges (black dotted lines) with the same size in the b direction are shown.

Fig. 2 (right panel) shows the calculated crystal habits superimposed to the observed crystal shapes. The trends obtained are summarized in Table 1 . The results indicate a decrease in $\mathrm{il}_{0}^{2} /\left(\mathrm{v}_{\mathrm{r}}+\mathrm{v}_{\mathrm{l}}\right)$ from 0.09 to 0.05 , but a stronger decrease in $v_{r} / v_{l}$ from 1 to 0.3 as $\Delta T$ increases and the crystals shape approaches square-like features. These results point towards a natural increase with $\Delta T$ of the step propagation rate with respect to the nucleation, but the strongest 
changes in the single crystals $\mathrm{I}_{\mathrm{b}} / \mathrm{l}_{\mathrm{a}}$ ratio are directly related to predicted changes in $v_{r} / v_{1}$ ratio.

At low $\Delta T\left(T_{C}=68{ }^{\circ} \mathrm{C}\right.$ in Table 1$), v_{r} / V_{l}$ is close to unity, and this ratio decreases with $\Delta T$ to a value of $V_{r} / V_{1}$ $=0.3$. Such low values of $\mathrm{v}_{\mathrm{r}} / \mathrm{v}_{\mathrm{l}}$ ratio directly provoke the emergence of closely square single crystals that are reported here for the first time.

As suggested by Scherbina and Ungar the difference between $v_{r}$ and $v_{l}$ arises from differences in molecular attachment rates, which are assumed to be related to the steric conditions of the chain attachment into the two non-equivalent niches on the right and lefts ends of the growth front [14-16]. In the PE orthorhombic lattice the two step angles at both ends of the growth front differ by $11^{\circ}$ (see Fig. 1). The adjacent chains attached on the left, separated by $\mathrm{I}_{0}$, have different setting angles, while those on the right, separated by $b_{0}$, have the same setting angle. The fitting results suggest that the left attachment steps of chains with different setting angles are preferred at $\Delta \mathrm{T}$ increases. For the time being, there are no explanations about why the attachment would be different when the setting angle of the chain differs.

\section{Conclusions}

Novel morphologies have been observed in solution grown single crystals bounded by curved $\{110\}$ lateral faces in short chain branched high molecular weight polyethylene. As the supercooling increases, the crystals tend to adopt an almost square-like morphology. The shapes of the crystals have been successfully predicted by applying the Shcherbina and Ungar model. The results can be rationalized by considering that a decrease in the step propagation ratio occurs as supercooling increases. This trend could be related to the different steric conditions of chain attachments in both sides of the growth $\{110\}$ face, due to the presence of the short chain branches.

\section{Acknowledgments}

The authors acknowledge funding support from the Spanish Ministerio de Economía y Competitividad (MINECO) - Projects MAT2012-36341 and MAT201453437-C2-P. Thanks are due to Repsol (Móstoles, Spain) for the supply of the sample.

\section{References}

[1] S.J. Organ, J.K. Hobbs and M.J. Miles, Reorganization and melting of polyethylene single crystals: Complementary TEM, DSC, and real-time AFM Studies, Macromolecules 37 (2004) 4562-4572.

[2] M. Tian, M. Dosière, S. Hocquet, P.J. Lemstra and J. Loos, Novel aspects related to nucleation and growth of solution grown polyethylene single crystals, Macromolecules 37 (2004) 1333-1341.
[3] J. Nakamura, M. Tsuji, A. Nakayama and A. Kawaguchi, Substrate-controlled reorganization of solution-grown polyethylene single crystals through partial melting, Macromolecules 41 (2008) 13581363.

[4] B. Zhang, J. Chen, M.C. Baier, S. Mecking, R. Reiter, R. Mülhaupt and G. Reiter, Molecular-weightdependent changes in morphology of solution-grown polyethylene single crystals, Macromol. Rapid Comm. 36 (2015) 181-189.

[5] S.J. Organ and A. Keller, Solution crystallization of polyethylene at high temperatures, J. Mat. Sci. 20 (1985) 1571-1585.

[6] S.Z.D. Cheng and J. Chen, Nonintegral and integral folding crystal growth in low-molecular mass poly (ethylene oxide) fractions. III. Linear crystal growth rates and crystal morphology, J. Polym. Sci. Part B.: Polym. Phys. 29 (1991) 311-327.

[7] A.J. Lovinger and H.D. Keith, Chain tilt in apoly(vinylidene fluoride), Macromolecules 29 (1996) 8541-8542.

[8] N. Sanandaji, L. Ovaskainen, M. Klein Gunnewiek, G.J. Vancso, M.S. Hedenqvist, S. Yu, L. Eriksson, S. V. Roth and U.W. Gedde, Unusual crystals of poly $(\varepsilon-$ caprolactone) by unusual crystallisation: The effects of rapid cooling and fast solvent loss on the morphology, crystal structure and melting, Polymer 54 (2013) 1497-1503.

[9] A. Toda, Growth mode and curved lateral habits of polyethylene single crystals, Faraday Discuss. 95 (1993) 129-143.

[10] G. Ungar and E.G.R. Putra, Asymmetric curvature of $\{110\}$ crystal growth faces in polyethylene oligomers, Macromolecules 34 (2001) 5180-5185.

[11] E.G.R. Putra and G. Ungar, In situ solution crystallization study of $\mathrm{n}-\mathrm{C} 246 \mathrm{H} 494$ : Self-poisoning and morphology of polymethylene crystals, Macromolecules 36 (2003) 5214-5225.

[12] G. Ungar, E.G.R. Putra, D.S.M. De Silva, M.A. Shcherbina and A.J. Waddon, The effect of selfpoisoning on crystal morphology and growth rates, Adv. Polym. Sci. 180 (2005) 45-87.

[13] J.F. Vega, N. Jargour, R. Núñez-Ramírez, G. Liu, D. Wang, M. Trujillo, A.J. Müller and J. MartínezSalazar, The influence of short-chain branching on the morphology and structure of polyethylene single crystals, J. Polym. Sci. Part B.: Polym. Phys. 53 (2015) 1751-1762.

[14] M.A. Shcherbina and G. Ungar, Solution of the growth equation for asymmetric crystal faces, Polymer 47 (2006) 5505-5512.

[15] M.A. Shcherbina and G. Ungar, Asymmetric curvature of growth faces of polymer crystals, Macromolecules 40 (2007) 402-405.

[16] M.A. Shcherbina and G. Ungar, Analysis of crystal habits bounded by asymmetrically curved faces: Polyethylene oligomers and poly(vinylidene fluoride), Polymer 48 (2007) 2087-2097.

[17] M. Aguilar, J.F. Vega, E. Sanz and J. MartinezSalazar, New aspects on the rheological behaviour of metallocene catalysed polyethylenes, Polymer 42 (2001) 9713-9721.

[18] M. Aguilar, J.F. Vega, A. Muñoz-Escalona and J. Martínez-Salazar, Temperature and branching dependence of surface extrusion instabilities in 
metallocene catalysed polyethylene, J. Mat. Sci. 37 (2003) 3415-3421.

[19] J. Otegui, J.F. Vega, S. Martín, V. Cruz, A. Flores, C. Domingo and J. Martínez-Salazar, The unit cell expansion of branched polyethylene as detected by Raman spectroscopy: An experimental and simulation approach, J. Mat. Sci. 42 (2007) 10461049.

[20] J.F. Vega, S. Martín, M.T. Expósito and J. MartínezSalazar, Entanglement network and relaxation temperature dependence of single-site catalyzed ethylene/1-hexene copolymers, J. Appl. Polym. Sci. 109 (2008) 1564-1569.

[21] S. Martín, J. Vega, M. Expósito, A. Flores and J. Martínez-Salazar, A three-phase microstructural model to explain the mechanical relaxations of branched polyethylene: a DSC, WAXD and DMTA combined study, Colloid Polym. Sci. 289 (2011) 257268.

[22] S. Martín, M.T. Expósito, J.F. Vega and J. MartínezSalazar, Microstructure and properties of branched polyethylene: Application of a three-phase structural model, J. Appl. Polym. Sci. 128 (2013) 1871-1878.

[23] F.M. Mirabella, Correlation of the melting behavior and copolymer composition distribution of ZieglerNatta-catalyst and single-site-catalyst polyethylene copolymers, J. Polym. Sci. Part B.: Polym. Phys. 39 (2001) 2800-2818.

[24] A. Toda and H. Kiho, Crystal growth of polyethylene from dilute solution: Growth kinetics of $\{110\}$ twins and diffusion-limited growth of single crystals, J. Polym. Sci.: Part B: Polym. Phys. 27 (1989) 53- 70.

[25] M.L. Mansfield, Solution of the growth equations of a sector of a polymer crystal including consideration of the changing size of the crystal, Polymer 29 (1988) 1755-1760.

[26] J.J. Point and D. Villers, Nucleation-controlled growth and normal growth: a unified view, J. Cryst. Growth 114 (1991) 228-238.

[27] A. Toda, Rounded lateral habits of polyethylene single crystals, Polymer 32 (1991) 771-780.

[28] S.T. Seto, Rep. Prog. Polym. Phys. Jpn. 7 (1964) 67-69.

[29] F.C. Frank, Nucleation-controlled growth on a onedimensional growth of finite length, J. Cryst. Growth 22 (1974) 233-236. 
Supplementary Material
Click here to download Supplementary Material: EPJ_Supplemental Information.tif

Supplementary Material
Click here to download Supplementary Material: EPJ_Supplemental Information.tif

Click here to download Supplementary Material: EPJ_Supplemental Information.tif

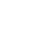

(1)

(1)

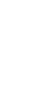
(1) (1)

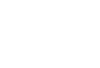

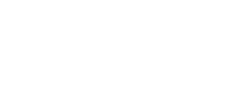
. . . . (1)

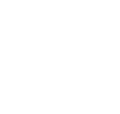

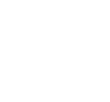
.

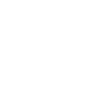

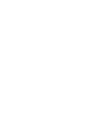

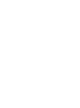
. . . . . 